Revue d'histoire de l'Amérique française

DA REVUE D.HISTOIRE DE L'AMÉRIQUE FRANÇAISE

\title{
Colonisation, climat et âge au baptême des Canadiens au XVII ${ }^{\mathbf{e}}$ siècle
}

\section{Hubert Charbonneau}

Volume 38, numéro 3, hiver 1985

Population et histoire

URI : https://id.erudit.org/iderudit/304282ar

DOI : https://doi.org/10.7202/304282ar

Aller au sommaire du numéro

Éditeur(s)

Institut d'histoire de l'Amérique française

ISSN

0035-2357 (imprimé)

1492-1383 (numérique)

Découvrir la revue

Citer cet article

Charbonneau, H. (1985). Colonisation, climat et âge au baptême des Canadiens au XVII siècle. Revue d'histoire de l'Amérique française, 38(3), 341-356.

https://doi.org/10.7202/304282ar d'utilisation que vous pouvez consulter en ligne.

https://apropos.erudit.org/fr/usagers/politique-dutilisation/ 


\title{
COLONISATION, CLIMAT ET ÂGE AU BAPTÊME DES CANADIENS AU XVII' ${ }^{\mathrm{e}}$ SIÈCLE
}

\author{
HUBERT CHARBONNEAU \\ Département de démographie \\ Université de Montréal
}

Au premier abord, la distinction entre date de naissance et date de baptême paraît sans intérêt. Comme ces deux dates sont généralement proches l'une de l'autre, on a tendance à les confondre, d'autant plus que les rédacteurs des anciens registres paroissiaux n'en mentionnent parfois qu'une seule. Quand on dispose du double renseignement, il est cependant possible de calculer l'âge au baptême par une simple soustraction. On obtient alors, au profit de la démographie historique, un excellent indicateur de la qualité des registres. Mais à l'époque où les missionnaires parcouraient le Saint-Laurent en canot, l'âge au baptême constitue un sujet qui déborde la simple question de l'enregistrement des actes. La seule comparaison des deux dates est en effet susceptible de nous éclairer sur les conditions de vie des pionniers du Canada.

Peu de travaux ont été consacrés à ce sujet, car le délai entre l'événement et l'acte était inférieur à 3 jours dans les pays catholiques d'Europe où les registres étaient autrefois bien tenus. En Angleterre, B. Midi Berry et R.S. Schofield ont toutefois observé, dans un nombre important de paroisses, une nette tendance à faire baptiser tardivement les enfants: si la médiane ne dépasse pas 8 jours au XVII ${ }^{\mathrm{e}}$ siècle, il apparaît qu'au moins un enfant sur deux était baptisé à plus de 25 jours à la fin du XVIII ${ }^{e}$ siècle $^{1}$. Hors du continent européen, les délais pouvaient s'allonger considérablement dans les pays de colonisation. C'est ainsi qu'au Brésil ou au Chili par exemple, quand une paroisse s'étendait sur d'immenses territoires, la dispersion du peuplement retardait le baptême, de sorte que la médiane atteignait fréquemment 10 jours ou plus ${ }^{2}$. $\mathrm{Au}$ Québec, un seul auteur, croyons-nous, s'est livré à une étude chiffrée

\footnotetext{
1 B. Midi Berry et R.S. Schofield, «Age at Baptism in Pre-Industrial England», Population Studies, 25, 3 (1971): 453-463.

2 René Salinas Meza, Un village colonial du Chili central: La Ligua. Société et population, 1700-1850, Thèse de Ph.D., Département d'histoire, Université de Montréal (1978), 117. - Ana Maria Burmester, La population de Curitiba au XVIII ${ }^{e}$ siècle, Thèse de Ph.D., Département de démographie, Université de Montréal (1981), 70.
} 
de la question: il s'agit de L.-E. Hamelin qui s'est intéressé à la vitalité religieuse d'une paroisse de Rouyn au $\mathrm{XX}^{\mathrm{e}}$ siècle ${ }^{3}$.

Nous disposons pour notre part des données du Programme de recherche en démographie historique 4 . Elles nous fournissent l'âge au baptême des Canadiens nés au XVII ${ }^{\mathrm{e}}$ siècle. Les dates de naissance et de baptême ont été relevées suivant des règles précises ${ }^{5}$ et le calcul de l'intervalle entre l'événement et l'acte a été fait à l'aide des procédés automatiques. Le recours à l'ordinateur, parce qu'il permet de multiplier les tableaux sans trop d'efforts, nous autorise à scruter ici avec le maximum d'exactitude certains aspects peu connus du comportement des premiers habitants de la colonie.

\section{Âge au baptême dans l'ensemble du Canada}

Les prêtres étaient-ils tenus d'inscrire les dates de naissance et de baptême dans l'acte de baptême? En 1539, l'Ordonnance civile de Villers-Cotterêts ne prescrit que la mention de la date de naissance ${ }^{6}$. Quelques années plus tard, le concile de Trente ne se réfère implicitement qu'à la seule date de baptême. Si la réglementation ecclésiastique exigea les deux dates dans le Rituale romanum de 1614, la réglementation civile n'en fit autant qu'à la fin du XVIII' siècle au Québec; c'est sans doute ce qui explique les lacunes de l'enregistrement à cet égard ${ }^{7}$. L'examen des registres des premières paroisses canadiennes montre en effet que les rédacteurs des actes obéissaient davantage à la hiérarchie catholique qu'au pouvoir civil et que les effets du concile de Trente se firent sentir longtemps.

Pour l'ensemble du XVII ${ }^{e}$ siècle, les deux dates sont connues avec précision dans plus de $75 \%$ des actes de baptême conservés jusqu'à nos jours, ce qui satisfait aux exigences de l'étude (tableau 1). C'est géné-

\footnotetext{
3 Louis-Edmond Hamelin, «Nombre de jours entre la naissance et le baptême dans une paroisse de Rouyn», Ad Usum Sacerdotum, 14, 3 (mars 1959): 73-76.

4 Le Programme de recherche en démographie historique (PRDH) procède, sous la direction d'Hubert Charbonneau et Jacques Légaré, à l'étude détaillée de la population québécoise depuis l'origine, avec l'aide financière du Conseil de recherches en sciences humaines du Canada, du Fonds FCAC et de l'Université de Montréal que nous remercions ici. Pour une description générale du programme de recherche voir: Jacques Légaré, «Le Programme de recherche en démographie historique de l'Université de Montréal: fondements, méthodes, moyens et résultats», Études canadiennes/Canadian Studies, 10 (juin 1981): 149-182. Nos remerciements s'adressent aussi à Pierre Beauchamp, Alain Bideau, Bertrand Desjardins, Yves Landry, Jacques Légaré et Pierre Rosa pour leur précieuse collaboration et leurs judicieux conseils.

${ }_{5}$ Programme de recherche en démographie historique, Du manuscrit à l'ordinateur: dépouillement des registres paroissiaux aux fins de l'exploitation automatique, publié sous la direction de Hubert Charbonneau et André LaRose (Québec, Archives nationales du Québec, 1980), «Études et recherches archivistiques», 3: 229 p.

6 Gérard Bouchard et André LaRose, «La réglementation du contenu des actes de baptême, mariage, sépulture au Québec, des origines à nos jours», Revue d' histoire de l'Amérique française, 30, 1 (juin 1976): 67-84.

Raymond Roy et Hubert Charbonneau, «Le contenu des registres paroissiaux canadiens du XVII ${ }^{\mathrm{e}}$ siècle», Revue d' histoire de l'Amérique française, 30, 1 (juin 1976): 88.
} 
ralement la date de naissance qui fait défaut, la date de baptême ne manquant qu'à de très rares occasions. Nous poserons plus loin la question de la représentativité de ces informations.

TABLEAU 1

Fréquence de l'inscription de l'année, du mois et du jour de l'acte et de l'événement dans les actes de baptême du Canada au XVII' siècle

\begin{tabular}{|c|c|c|c|c|c|}
\hline \multirow{3}{*}{$\begin{array}{l}\text { Éléments connus } \\
\text { de la date de } \\
\text { baptême }\end{array}$} & \multicolumn{5}{|c|}{ Éléments connus de la date de naissance } \\
\hline & \multicolumn{5}{|c|}{ (nombre absolu) } \\
\hline & $\begin{array}{l}\text { Année } \\
\text { mois, jour }\end{array}$ & $\begin{array}{l}\text { Année } \\
\text { et mois }\end{array}$ & $\begin{array}{c}\text { Année } \\
\text { seulement }\end{array}$ & $\begin{array}{l}\text { Aucun } \\
\text { élément }\end{array}$ & Ensemble \\
\hline Année, mois, jour & $13517 *$ & 36 & 0 & 3963 & 17516 \\
\hline Année et mois & 7 & 10 & 1 & 34 & 52 \\
\hline Année seulement & 3 & 0 & 3 & 34 & 40 \\
\hline Aucun élément & 112 & 6 & 0 & 0 & 118 \\
\hline \multirow[t]{3}{*}{ Ensemble } & 13639 & 52 & 4 & 4031 & $\overline{17726}$ \\
\hline & \multicolumn{5}{|c|}{ (nombre relatif) } \\
\hline & $\begin{array}{l}\text { Année } \\
\text { mois, jour }\end{array}$ & $\begin{array}{l}\text { Année } \\
\text { et mois }\end{array}$ & $\begin{array}{c}\text { Année } \\
\text { seulement }\end{array}$ & $\begin{array}{l}\text { Aucun } \\
\text { élément }\end{array}$ & Ensemble \\
\hline Année, mois, jour & 76,3 & 0,2 & 0,0 & 22,3 & 98,8 \\
\hline Année et mois & 0,1 & 0,1 & 0,0 & 0,2 & 0,3 \\
\hline Année seulement & 0,0 & 0,0 & 0,0 & 0,2 & 0,2 \\
\hline Aucun élément & 0,6 & $\underline{0,0}$ & 0,0 & 0,0 & 0,7 \\
\hline Ensemble & 77,0 & $\overline{0,3}$ & $\overline{0,0}$ & 22,7 & 100,0 \\
\hline
\end{tabular}

* À l'exclusion de dix adultes originaires de la Nouvelle-Angleterre.

Sur les 13517 enfants pris en compte l'âge médian au baptême n'est que de 1,5 jour, mais l'intervalle moyen s'établit à 4,7 jours, ce qui est élevé en comparaison de la situation qui prévalait alors en France. Près du tiers des nouveau-nés canadiens étaient baptisés le jour de leur naissance et les deux tiers au plus tard le lendemain (tableau 2). Un quart des enfants étaient conduits à l'église après le surlendemain de leur naissance, ce qui mérite d'être souligné. La moitié de ces derniers recevaient le baptême après leur première semaine de vie. Environ 3\% des enfants étaient baptisés à 1 mois ou plus. On s'attendrait néanmoins à de plus longs délais encore, compte tenu des caractéristiques géographiques de la colonie.

L'examen détaillé des 14 actes relatifs aux enfants baptisés à 7 mois et plus montre par ailleurs qu'il s'agit surtout d'enfants de familles nobles ou en vue, les autres étant des illégitimes, des enfants nés en des 
lieux éloignés ou des ondoyés dont on s'explique mal qu'ils aient été baptisés si tardivement.

\section{Âge au baptême par période}

Il convient ici de distinguer la période antérieure à 1680 , puisque l'année 1679 correspond à la fois à l'organisation d'un grand nombre de paroisses et au moment où le Conseil souverain a entériné au Canada l'ordonnance de Louis XIV (1667) traitant des registres paroissiaux. Durant la période 1621-1679, les deux dates sont moins souvent connues que par la suite: $71,2 \%$ contre $79,3 \%$; ce qui signifie que les intervalles indéterminés ne se répartissent pas dans le temps comme ceux dont le calcul est possible. Mais si on suppose que les intervalles inconnus se distribuent comme les autres à l'intérieur de chaque période décennale, on constate que la moyenne d'ensemble s'élève à 4,69 au lieu de 4,71, ce qui tend à montrer que l'effet des variations de l'enregistrement dans le temps est négligeable.

TABLEAU 2

Distribution des baptisés suivant l'âge au baptême dans l'ensemble du Canada au XVII' ${ }^{\mathrm{e}}$ siècle

\begin{tabular}{lccc}
\hline Âge au baptême & $\begin{array}{c}\text { Nombre absolu } \\
\text { de baptisés }\end{array}$ & $\begin{array}{c}\text { Nombre relatif } \\
\text { de baptisés }\end{array}$ & $\begin{array}{c}\text { Nombre relatif } \\
\text { cumulé de baptisés }\end{array}$ \\
\hline 0 jour & 4247 & 31,42 & 31,42 \\
1 jour & 4665 & 34,51 & 65,93 \\
2 jours & 1251 & 9,26 & 75,19 \\
3 jours & 682 & 5,05 & 80,24 \\
4 jours & 421 & 3,11 & 83,35 \\
5 jours & 292 & 2,16 & 85,51 \\
6 jours & 249 & 1,84 & 87,35 \\
$7-13$ jours & 789 & 5,84 & 93,19 \\
$14-20$ jours & 325 & 2,40 & 95,59 \\
21 jours à 1 mois & 202 & 1,50 & 97,09 \\
1 mois & 237 & 1,75 & 98,84 \\
2 mois & 72 & 0,53 & 99,37 \\
3 mois & 25 & 0,19 & 99,56 \\
4 mois & 19 & 0,14 & 99,70 \\
5 mois & 16 & 0,12 & 99,82 \\
6 mois & 11 & 0,08 & 99,90 \\
7 mois & 3 & 0,02 & 99,92 \\
8 mois & 1 & 0,01 & 99,93 \\
9 mois & 0 & 0,00 & 99,93 \\
10 mois & 3 & 0,02 & 99,95 \\
11 mois & 2 & 0,01 & 99,96 \\
1 an & 4 & 0,03 & 99,99 \\
2 ans & 1 & 0,01 & 100,00 \\
Tous âges & 1317 & 100,00 & \\
\hline & & &
\end{tabular}


Il ressort du tableau 3 que les baptêmes étaient davantage retardés à l'origine du pays, quand le peuplement était plus dispersé, les communications plus difficiles, l'organisation paroissiale peu développée. De même l'inscription des deux dates a constamment progressé tout au long du siècle. Aucun intervalle ne peut être calculé à propos des actes d'avant 1640, surtout en raison de la perte du premier registre de Québec. De 1640 à 1650 les nombres sont petits et incertains et le peuplement est relativement concentré. La décennie 1650-59 se distingue par un âge moyen au baptême très élevé, le tiers des enfants étant amenés à l'église après la première semaine de vie. L'arrivée du premier évêque en 1659 se fait ensuite sentir, car les longs intervalles diminuent brus-

TABLEAU 3

Âge au baptême suivant la période

\begin{tabular}{|c|c|c|c|c|c|c|c|}
\hline \multirow[b]{3}{*}{ Période } & \multirow{3}{*}{$\begin{array}{l}\text { Nombre total } \\
\text { de baptisés }\end{array}$} & \multirow{3}{*}{$\begin{array}{c}\text { Âge au baptême } \\
\text { connu pour } 100 \\
\text { baptisés }\end{array}$} & \multicolumn{5}{|c|}{ Âge au baptême (en jours) } \\
\hline & & & \multirow[b]{2}{*}{ Moyen } & \multicolumn{4}{|c|}{ Percentiles } \\
\hline & & & & $25 \%$ & $\begin{array}{c}50 \% \\
\text { (médiane) }\end{array}$ & $75 \%$ & $90 \%$ \\
\hline $1620-29$ & 6 & 0 & - & - & - & - & - \\
\hline $1630-39$ & 47 & 0 & - & - & - & - & - \\
\hline $1640-49$ & 202 & 36,1 & 3,5 & 0,6 & 1,2 & 2,6 & 15,0 \\
\hline $1650-59$ & 691 & 68,5 & 18,1 & 1,1 & 2,6 & 16,0 & 54,9 \\
\hline $1660-69$ & 1746 & 66,7 & 5,0 & 1,1 & 1,9 & 4,0 & 9,1 \\
\hline $1670-79$ & 3917 & 76,4 & 6,7 & 1,1 & 2,1 & 6,2 & 16,9 \\
\hline $1680-89$ & 4537 & 80,4 & 4,3 & 0,9 & 1,6 & 3,1 & 10,1 \\
\hline $1690-99$ & 6580 & 78,6 & 2,3 & 0,6 & 1,2 & 1,8 & 3,5 \\
\hline $1620-59$ & 946 & 57,8 & 16,2 & 1,0 & 2,0 & 13,9 & 48,6 \\
\hline $1660-79$ & 5663 & 73,3 & 6,3 & 1,1 & 2,0 & 5,3 & 13,8 \\
\hline $1680-99$ & 11117 & 79,3 & 3,1 & 0,7 & 1,4 & 2,1 & 5,6 \\
\hline $1620-99$ & 17726 & 76,3 & 4,7 & 0,8 & 1,5 & 3,0 & 10,2 \\
\hline
\end{tabular}

quement après cette date. Il faut dire que de nouveaux registres apparaissent sur la côte de Beaupré et à l'île d'Orléans. On observe pourtant une augmentation de l'âge au baptême pour la décennie 1670-79, probablement par suite de l'extension rapide du peuplement. C'est sans doute pourquoi Monseigneur de Laval s'est employé à rappeler le contenu de son ordonnance du 29 mars $1664^{8}$ à propos du délai entre naissance et baptême. Le 5 février 1677, il ordonne aux parents de porter leur enfant à l'église aussitôt après la naissance, le péril de mort seul légitimant l'ondoiement à la maison ${ }^{9}$. En cas d'infraction, les parents

8 «Ordonnance sur l'administration du sacrement de baptême» (29 mars 1664) et «Ordonnance pour l'administration du sacrement de baptême» (5 février 1677), dans H. Têtu et C.-O. Gagnon, édit., Mandements, lettres pastorales et circulaires des évêques de Québec, volume premier (Québec, Côté, 1887): 161-162 et 100-101.

${ }_{9}$ Raymond Douville, «Baptême», Mémoires de la Société généalogique canadiennefrançaise, III, 1 (janvier 1948): 16-17. 
devaient être ipso facto exclus de l'Église pour un mois, la récidive entraînant même l'excommunication.

Pour déterminer ce qui empêchait les colons de se conformer aux exigences du prélat, il convient d'examiner maintenant les distributions d'âge au baptême en fonction des lieux de peuplement de façon à mettre en lumière le rôle des difficultés de communication et de l'éloignement de l'église. L'influence des rédacteurs d'acte ne peut être négligée non plus et il se peut que certains prêtres aient été plus rigoureux que d'autres au chapitre qui nous intéresse ici. Nous avons d'ailleurs déjà relevé des différences à propos de l'enregistrement des actes entre les diverses communautés et le clergé séculier ${ }^{10}$. L'effet des saisons et notamment des rudes hivers doit être également étudié. En ce qui concerne les variations suivant le sexe, disons tout de suite qu'il n'y en a pas, même s'il y a tout lieu de croire que les garçons couraient de plus forts risques de décès que les filles durant les premiers jours de vie.

\section{Âge au baptême par gouvernement}

Les intervalles moyens et médians varient assez peu d'un gouvernement à l'autre dans l'ensemble du siècle (tableau 4). Celui de Montréal présente à la fois la moyenne la plus élevée et la médiane la plus basse. Il faut dire que la qualité de l'information n'est vraiment satisfaisante que dans le gouvernement de Québec. Peut-être l'influence de l'évêque diminuait-elle avec l'éloignement, bien que les deux dates aient déjà été relevées avec régularité avant même l'arrivée de celui-ci dans le groupe de paroisses rattachées à Québec. Les rédacteurs d'actes étaient par contre des séculiers, principalement dans le gouvernement de Québec, alors que les Sulpiciens ont rédigé trois actes sur quatre

TABLEAU 4

Âge au baptême par gouvernement

\begin{tabular}{|c|c|c|c|c|c|c|}
\hline \multirow[b]{3}{*}{ Gouvernement } & \multirow{3}{*}{$\begin{array}{c}\text { Âge au baptême } \\
\text { connu pour } 100 \\
\text { baptisés }\end{array}$} & \multicolumn{5}{|c|}{ Âge au baptême (en jours) } \\
\hline & & \multirow[b]{2}{*}{ Moyen } & \multicolumn{4}{|c|}{ Percentiles } \\
\hline & & & $25 \%$ & $\begin{array}{c}50 \% \\
\text { (médiane) }\end{array}$ & $75 \%$ & $90 \%$ \\
\hline Québec & 88,8 & 4,5 & 0,8 & 1,6 & 3,3 & 10,7 \\
\hline Trois-Rivières & 59,4 & 4,3 & 0,9 & 1,6 & 3,0 & 9,6 \\
\hline Montréal & 54,4 & 5,0 & 0,6 & 1,3 & 2,2 & 6,8 \\
\hline Ensemble & 76,3 & 4,7 & 0,8 & 1,5 & 3,0 & 10,2 \\
\hline
\end{tabular}

10 Raymond Roy et Hubert Charbonneau, loc. cit., 96. 
dans celui de Montréal avant $1700^{11}$. Dans ce dernier gouvernement, la date de naissance n'est pratiquement jamais relevée avant la décennie 1670-79. La situation se corrige par la suite et on peut y voir la conséquence des interventions répétées de l'autorité civile ou religieuse. De même, l'âge au baptême diminue partout vers la fin du siècle et l'écart entre gouvernements a tendance à s'estomper.

\section{Âge au baptême suivant l'habitat}

Baptisait-on les enfants plus rapidement à la ville qu'à la campagne? L'information à ce sujet est meilleure en milieu rural qu'en milieu urbain (tableau 5), ce qui provient essentiellement du fait que les Sulpiciens indiquaient rarement la date de naissance dans les actes de baptême du registre de Montréal. Contre toute attente, l'intervalle moyen entre l'événement et l'acte était aussi plus long à la ville si on s'en tient à l'ensemble du XVII' siècle. Environ trois baptêmes sur quatre avaient pourtant lieu avant la fin du deuxième jour de vie en ville contre à peine trois sur cinq à la campagne. Aussi la médiane se révèlet-elle plus courte en milieu urbain. Quelques longs intervalles influent de façon déterminante sur la moyenne: on observe en effet un peu plus de délais supérieurs à 1 mois dans les registres urbains, notamment à l'origine du pays quand les missionnaires y consignaient des actes relatifs à des événements dispersés le long du Saint-Laurent. La comparaison ne prend toute sa signification qu'après 1680 , alors que moyennes et médianes se font plus courtes à la ville qu'à la campagne. C'est

TABLEAU 5

Âge moyen et âge médian au baptême suivant l'habitat par période

\begin{tabular}{|c|c|c|c|c|c|c|c|c|}
\hline \multirow[t]{2}{*}{ Période } & \multicolumn{2}{|c|}{$\begin{array}{c}\text { Nombre total } \\
\text { de baptisés }\end{array}$} & \multicolumn{2}{|c|}{$\begin{array}{c}\text { Âge au baptême } \\
\text { connu pour } 100 \\
\text { baptisés }\end{array}$} & \multicolumn{2}{|c|}{$\begin{array}{c}\text { Âge moyen } \\
\text { au baptême } \\
\text { (en jours) }\end{array}$} & \multicolumn{2}{|c|}{$\begin{array}{l}\text { Âge médian } \\
\text { au baptême } \\
\text { (en jours) }\end{array}$} \\
\hline & Ville* & Campagne & Ville & Campagne & Ville & Campagne & Ville & Campagne \\
\hline $1640-49$ & 162 & 40 & 35,8 & 37,5 & 4,3 & 0,5 & 1,4 & 0,7 \\
\hline $1650-59$ & 541 & 150 & 79,7 & 26,7 & 19,0 & 8,2 & 2,9 & 1,7 \\
\hline $1660-69$ & 1030 & 716 & 57,7 & 79,3 & 5,1 & 4,9 & 1,6 & 2,3 \\
\hline $1670-79$ & 1980 & 1937 & 73,5 & 79,1 & 6,7 & 6,7 & 1,8 & 2,7 \\
\hline $1680-89$ & 1154 & 3383 & 67,6 & 84,7 & 1,8 & 4,9 & 1,1 & 1,8 \\
\hline $1690-99$ & 2244 & 4336 & 78,1 & 78,7 & 1,7 & 2,7 & 0,9 & 1,3 \\
\hline $1620-99$ & 7159 & 10567 & 70,8 & 79,8 & 5,0 & 4,3 & 1,3 & 1,7 \\
\hline
\end{tabular}

* Les paroisses de Québec et de Montréal sont considérées comme urbaines, toutes les autres comme rurales, y compris Trois-Rivières.

1I Raymond Roy, Yves Landry et Hubert Charbonneau, "Quelques comportements des Canadiens au XVII ${ }^{\mathrm{e}}$ siècle d'après les registres paroissiaux», Revue d'histoire de l'Amérique française, 31,1 (juin 1977): 51 . 
d'ailleurs à cette époque que la date de naissance apparaît de plus en plus fréquemment dans le registre de Montréal.

\section{Âge au baptême par paroisse}

À l'échelon paroissial, les registres de Notre-Dame-de-Québec occupent une place à part, puisqu'ils comptent plus de $25 \%$ des actes de baptême du XVII ${ }^{\mathrm{e}}$ siècle. L'enregistrement y est fort heureusement de grande qualité et plus de $91 \%$ des actes de baptême contiennent les deux dates requises. Si on limite l'étude aux 28 autres registres contenant au moins 100 actes avec les deux dates avant 1700 , on trouve neuf paroisses où l'âge au baptême est connu pour au moins $90 \%$ des nouveaunés. Ce sont surtout des paroisses des environs de Québec: Neuville, Beauport, Sainte-Famille de l'île-d'Orléans, Charlesbourg et Lauzon, auxquelles s'ajoutent Rivière-Ouelle, Contrecoeur, Boucherville et Repentigny (voir annexe). À l'opposé, Montréal a le pire dossier en matière d'inscription des deux dates avec moins de $34 \%$ d'âge au baptême connu. Seule Pointe-aux-Trembles, qui était également desservie par les Sulpiciens, offre une proportion comparable.

Les intervalles moyens et médians sont les plus élevés dans les paroisses qui desservaient par voie de mission des localités privées de prêtres: Sorel, Contrecoeur, Rivière-Ouelle où l'âge médian au baptême est compris entre 5 et 6 jours et Québec durant la décennie 165059 où l'âge médian ne dépasse pas 3 jours en dépit d'une moyenne de près de 22 jours. Les délais les plus brefs se retrouvent par contre à Charlesbourg et Beauport de même qu'à Laprairie où moyennes et médianes oscillent autour d'une journée.

Dans l'ensemble, il ne paraît pas y avoir de lien entre l'âge au baptême et la qualité de l'information, car les intervalles ne varient pas de façon significative en fonction de la proportion d'âges au baptême connus dans une paroisse. Aussi bien sur le plan géographique que chronologique c'est une question de clergé avant tout.

\section{Effet du jour de naissance sur l'âge au baptême}

L'étude du mouvement quotidien des actes montre qu'il y a d'autant moins de baptêmes qu'on se rapproche du dimanche (tableau 6) ${ }^{12}$. Comme 20\% des baptêmes avaient lieu le dimanche, on peut penser que le fait de naître un mercredi par exemple pouvait reporter la cérémonie de quelques jours. En réalité l'attraction dominicale a assez peu d'effet sur les indices relatifs à l'âge au baptême: les âges moyen et médian sont plus faibles le lundi qui est le jour de la semaine le plus éloigné du dimanche suivant (tableau 7). La diminution de l'intervalle, du lundi au samedi, n'est perceptible qu'à compter du vendredi pour le $75^{\mathrm{e}}$ percentile seulement.

12 Ibid., 63. 
TABLEAU 6

Distribution des baptêmes suivant le jour de la semaine et l'âge des baptisés

\begin{tabular}{|c|c|c|c|c|c|c|c|c|c|}
\hline \multirow{2}{*}{$\begin{array}{c}\text { Âge } \\
\text { des baptisés } \\
\text { (en jour) }\end{array}$} & \multicolumn{8}{|c|}{ Jour du baptême } & \multirow{2}{*}{$\begin{array}{c}\text { Nombres } \\
\text { absolus } \\
\text { de baptêmes }\end{array}$} \\
\hline & Dimanche & Lundi & Mardi & Mercredi & Jeudi & Vendredi & Samedi & Ensemble & \\
\hline 0 & 167 & 158 & 122 & 137 & 141 & 142 & 133 & 1000 & 4247 \\
\hline 1 & 177 & 140 & 143 & 148 & 135 & 136 & 121 & 1000 & 4665 \\
\hline 2 & 237 & 128 & 135 & 127 & 141 & 106 & 126 & 1000 & 1251 \\
\hline 3 & 284 & 150 & 110 & 92 & 129 & 109 & 126 & 1000 & 682 \\
\hline 4 & 304 & 133 & 97 & 119 & 126 & 93 & 128 & 1000 & 421 \\
\hline 5 & 325 & 154 & 86 & 127 & 137 & 79 & 92 & 1000 & 292 \\
\hline 6 & 293 & 88 & 145 & 149 & 108 & 84 & 133 & 1000 & 249 \\
\hline 7 et plus & 282 & 142 & 132 & 121 & 120 & 108 & 95 & 1000 & 1710 \\
\hline Tous âges & 207 & 144 & 130 & 135 & 135 & 127 & 122 & 1000 & 13517 \\
\hline $\begin{array}{l}\text { Nombres absolus } \\
\text { de baptisés }\end{array}$ & 2802 & 1952 & 1758 & 1825 & 1820 & 1711 & 1649 & 13517 & \\
\hline
\end{tabular}

TABLEAU 7

Âge au baptême suivant le jour de naissance

\begin{tabular}{|c|c|c|c|c|c|}
\hline \multirow{3}{*}{$\begin{array}{c}\text { Jour de } \\
\text { naissance }\end{array}$} & \multicolumn{5}{|c|}{ Âge au baptême (en jours) } \\
\hline & \multirow[b]{2}{*}{ Moyen } & \multicolumn{4}{|c|}{ Percentiles } \\
\hline & & $25 \%$ & $\begin{array}{c}50 \% \\
\text { (médiane) }\end{array}$ & $75 \%$ & $90 \%$ \\
\hline Dimanche & 4,8 & 0,7 & 1,5 & 2,9 & 12,6 \\
\hline Lundi & 3,8 & 0,7 & 1,4 & 2,7 & 8,1 \\
\hline Mardi & 4,9 & 0,9 & 1,6 & 3,1 & 9,5 \\
\hline Mercredi & 4,5 & 0,8 & 1,5 & 3,5 & 9,1 \\
\hline Jeudi & 4,6 & 0,8 & 1,6 & 3,4 & 9,9 \\
\hline Vendredi & 5,2 & 0,8 & 1,6 & 2,9 & 10,2 \\
\hline Samedi & 4,4 & 0,9 & 1,5 & 2,7 & 10,5 \\
\hline Ensemble & 4,7 & 0,8 & 1,5 & 3,0 & 10,2 \\
\hline
\end{tabular}

Le baptême avait plus souvent lieu le jour même de la naissance le dimanche et le lundi et moins fréquemment que les autres jours le mardi et le samedi. Les enfants nés le mardi et surtout le samedi recevaient davantage le baptême le lendemain de leur naissance que ceux qui naissaient les autres jours de la semaine. Nous nous expliquons mal le report du mardi au mercredi de baptêmes d'enfants nés principalement dans les paroisses rurales. Mais nous comprenons mieux que les nouveau-nés du vendredi aient été ceux qui étaient le plus fréquemment amenés à l'église le surlendemain de leur naissance. 
Les nouveau-nés du dimanche étaient moins souvent baptisés que les autres à moins d'une semaine. C'est sans doute que la cérémonie était reportée au dimanche suivant leur naissance pour plusieurs d'entre eux. L'attraction exercée par le jour dominical atteint finalement les baptisés de tous âges, mais davantage ceux qui recevaient le sacrement à 3 jours et plus, effet camouflé par l'âge moyen en raison de la pondération exercée par le grand nombre de baptisés à 0 jour et à 1 jour. Malgré tout, les pressions de l'évêque à la promptitude atténuaient sûrement les velléités que l'on pouvait avoir de faire baptiser le jour du Seigneur.

\section{Effet du climat sur l'âge au baptême}

Malgré leur bonne volonté, les colons se voyaient parfois dans l'impossibilité d'obtempérer aux exigences de l'autorité ecclésiastique à cause de la rigueur de l'hiver canadien. Lors des tempêtes de neige ou des grands froids, les parents pouvaient hésiter à exposer un nouveauné même sur des distances assez courtes. Il suffit pour s'en convaincre de s'en remettre au texte des actes. Voici, par exemple, un extrait révélateur du registre de Trois-Rivières:

Le $27 \mathrm{e}$ jour de janvier de l'an 1692 par moy prêtre curé soussigné a été baptisé en la maison à cause du froid les cérémonies diférées jusqu'au tems doux un enfant masle né le même jour du mariage de françois vanasses et de Jeanne Fourier les cérémonies supplées le 13 du mois suivant de la dite année, assiste comme parein Etienne Gélinas m. marguerite Aubuchon fait ce tresième cy dessus marqué. A Maudoux ptre.

Raymond Douville cite des actes du registre de Batiscan du début du XVIII ${ }^{\mathrm{e}}$ siècle qui illustrent bien l'effet du climat sur la circulation et sur l'allongement de l'intervalle entre la naissance et le baptême ${ }^{13}$ :

Le 22 janvier 1710 je soussigné prestre curé de Batiscan ay baptisé un fils né du légitime mariage de Jean Brouillet et de Magdeleine Ricart, né le treize du mesme mois, les parens n'ayant pu l'apporter plus tost à l'Église à cause du froid et des mauvais chemins...

Le 7 décembre de l'an 1710, dans la coste de St-Pierre qui est à l'opposé de Batiscan est née une fille du mariage légitime de Pierre Masson et de Catherine François et a été ondoyée le même jour par René Pinot, et le 23 avril 1711 ils l'ont apportée à Batiscan, ne l'ayant pu plus tôt à cause que la rivière n'estant pas prise, ils ne pouvaient traverser...

L'observation statistique confirme ces brèves mais éloquentes mentions (tableau 8). La comparaison avec la température moyenne

13 Raymond Douville, loc. cit., 16-17. 
mensuelle montre une relation inversement proportionnelle à la fréquence des baptêmes tardifs (figure 1). Presque deux fois plus grand en janvier qu'en juillet, l'âge moyen au baptême diminue au coeur de l'hiver, au moment où la circulation peut se faire sur la neige, pour ensuite remonter lors de la fonte printanière. Le relèvement de la courbe en automne ne peut s'expliquer cependant par des considérations de ce genre; les travaux saisonniers seraient-ils en cause? Octobre à février sont dans l'ensemble au-dessus de la moyenne et mars à septembre en dessous.

TABLEAU 8

Âge au baptême suivant le mois de naissance

\begin{tabular}{|c|c|c|c|c|c|c|}
\hline \multirow{3}{*}{$\begin{array}{l}\text { Mois de } \\
\text { naissance }\end{array}$} & \multirow{3}{*}{$\begin{array}{l}\text { Nombre de } \\
\text { baptêmes à } \\
3 \text { jours et plus } \\
\text { (pour 100) }\end{array}$} & \multicolumn{5}{|c|}{ Âge au baptême (en jours) } \\
\hline & & \multirow[b]{2}{*}{ Moyen } & \multicolumn{4}{|c|}{ Percentiles } \\
\hline & & & $25 \%$ & $\begin{array}{c}50 \% \\
\text { (médiane) }\end{array}$ & $75 \%$ & $90 \%$ \\
\hline Janvier & 30,5 & 6,2 & 0,9 & 1,7 & 3,9 & 12,7 \\
\hline Février & 26,7 & 4,9 & 0,9 & 1,6 & 3,3 & 10,5 \\
\hline Mars & 25,9 & 4,0 & 0,8 & 1,6 & 3,2 & 9,9 \\
\hline Avril & 25,5 & 4,4 & 0,8 & 1,6 & 3,1 & 11,0 \\
\hline Mai & 26,7 & 3,7 & 0,8 & 1,5 & 3,3 & 11,4 \\
\hline Juin & 20,6 & 3,6 & 0,6 & 1,4 & 2,5 & 6,9 \\
\hline Juillet & 18,6 & 3,4 & 0,7 & 1,4 & 2,2 & 6,2 \\
\hline Août & 21,9 & 3,9 & 0,7 & 1,4 & 2,7 & 9,5 \\
\hline Septembre & 25,1 & 4,1 & 0,8 & 1,6 & 3,0 & 10,6 \\
\hline Octobre & 27,4 & 5,5 & 0,9 & 1,6 & 3,4 & 11,7 \\
\hline Novembre & 23,7 & 5,4 & 0,8 & 1,5 & 2,9 & 9,7 \\
\hline Décembre & 22,5 & 5,7 & 0,8 & 1,5 & 2,7 & 8,8 \\
\hline Ensemble & 24,8 & 4,6 & 0,8 & 1,5 & 3,0 & 10,2 \\
\hline
\end{tabular}

La médiane ne varie guère en fonction des saisons. Les mois où l'on baptisait le moins le jour de la naissance étaient janvier $(27,4 \%)$ et février, ceux où l'on baptisait le plus étant juin $(38,7 \%)$ et juillet. De septembre à avril, un nouveau-né avait plus de chances d'aller à l'église le second jour que le premier. La probabilité de recevoir le baptême dans les trois jours était la plus élevée de juin à août, ce dernier mois étant suivi de près par décembre, alors que la proportion de février l'emporte sur celle d'octobre. Les écarts mensuels ne résultent pas exclusivement des longs intervalles: ceux-ci sont par exemple plus fréquents en mai qu'en décembre en dépit d'un âge moyen au baptême nettement moins élevé. Mais le climat n'explique pas tout non plus. 
FIGURE 1

Âge au baptême suivant le mois de naissance
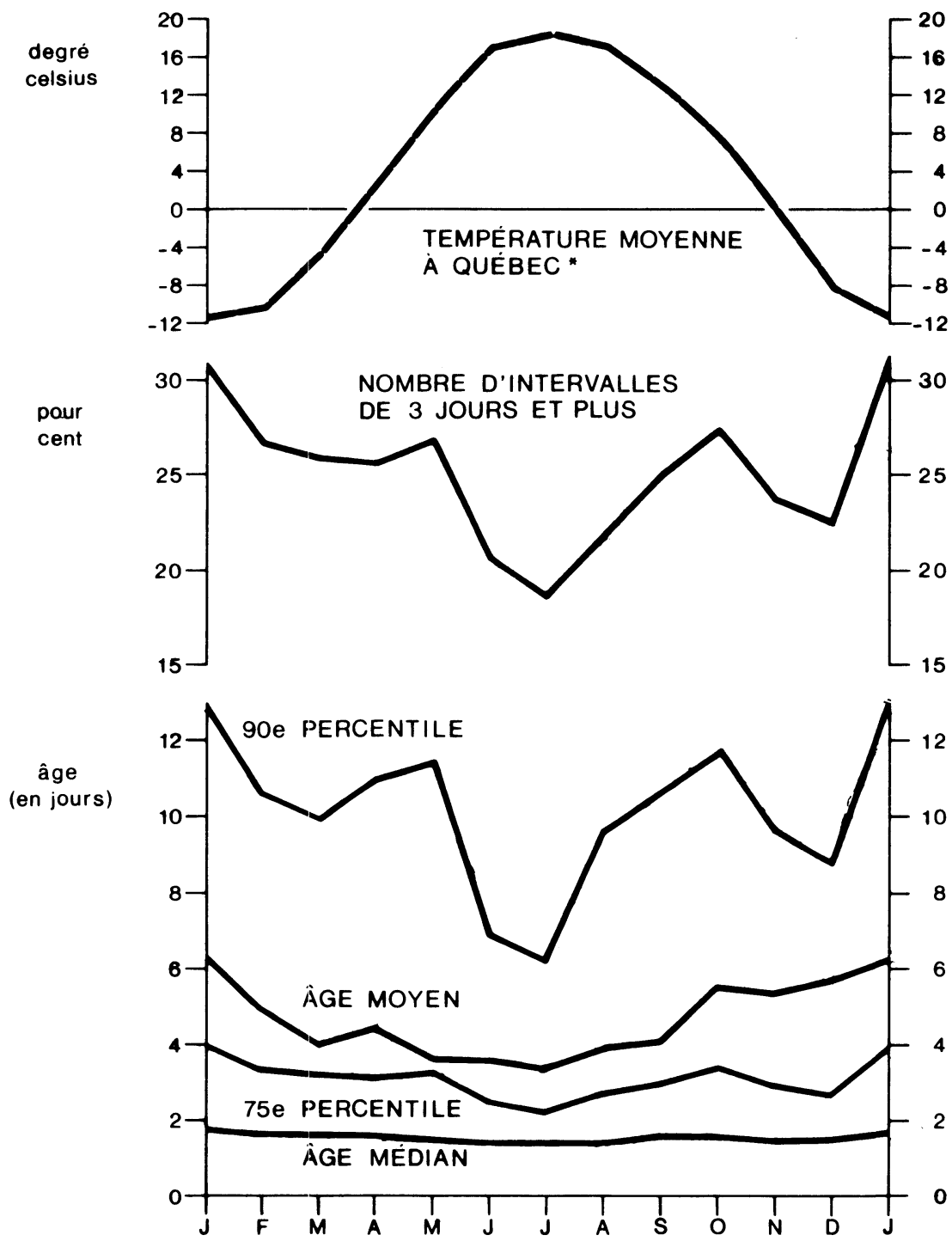

* Sources: Service de la Météorologie, Ministère des Richesses naturelles, Québec (Données de 1900 à 1976) 
L'observation par période montre qu'avant 1680 seulement $52 \%$ des naissances de janvier étaient suivies de baptêmes dans les trois jours contre $70 \%$ au mois de juillet. D'une grande période à l'autre, la chute de l'âge moyen au baptême est très nette en hiver, mais également au coeur de l'été (tableau 9). Durant la période la plus ancienne, septembre présente la plus faible moyenne et janvier la plus haute; après 1680 , on retrouve plutôt juillet et octobre. On remarque une certaine résistance automnale à la diminution des intervalles, ce qui restreint quelque peu l'étalement marqué de la distribution mensuelle des âges moyens.

TABLEAU 9

Âge moyen au baptême par grande période suivant le mois de naissance

\begin{tabular}{lccc}
\hline \multirow{2}{*}{$\begin{array}{c}\text { Mois de } \\
\text { naissance }\end{array}$} & \multicolumn{2}{c}{ Âge moyen au baptême (en jours) } & \multirow{2}{*}{$\begin{array}{c}\text { Diminution } \\
\text { (en \%) }\end{array}$} \\
\cline { 2 - 3 } & $1620-1679$ & $1680-1699$ & -67 \\
\hline Janvier & 11,3 & 3,8 & -73 \\
Février & 9,4 & 2,6 & -56 \\
Mars & 6,5 & 2,9 & -66 \\
Avril & 7,5 & 2,6 & -53 \\
Mai & 5,8 & 2,8 & -60 \\
Juin & 6,3 & 2,6 & -67 \\
Juillet & 6,1 & 2,1 & -52 \\
Août & 5,9 & 2,9 & -37 \\
Septembre & 5,5 & 3,5 & -42 \\
Octobre & 7,5 & 4,4 & -47 \\
Novembre & 7,8 & 4,2 & -55 \\
Décembre & 8,9 & 4,1 & -58 \\
Ensemble & 7,5 & 3,2 & \\
\hline
\end{tabular}

Dans l'ensemble du XVII ${ }^{\mathrm{e}}$ siècle, l'influence des saisons paraît plus marquée à la ville qu'à la campagne si l'on s'en tient à l'étude de l'âge moyen. Mais la comparaison entre habitats rural et urbain ne prenant tout son sens qu'à la fin du siècle, on constate qu'après 1680 les effets négatifs de l'hiver disparaissent complètement à la ville et qu'au contraire la proportion de baptêmes célébrés après les trois premiers jours de vie s'y révèle très faible de novembre à avril (tableau 10). À la campagne par contre, la fraction des baptisés de 3 jours et plus est cinq fois plus forte qu'en milieu urbain et l'influence du climat reste apparemment importante, sauf en décembre où le comportement se compare à celui des mois d'été. L'allongement des intervalles en octobre se retrouve, quel que soit l'habitat, peut-être en raison des activités et des déplacements qui précédaient le retour de la saison froide.

À l'échelle régionale, signalons le comportement exceptionnel des ruraux des environs de Québec. Ces derniers font baptiser encore plus 
vite qu'à la ville après 1680 , ce qui n'interdit pas toute influence du climat; néanmoins, il semble que dans ces paroisses on se soit affranchi avant la fin du siècle des difficultés propres aux débuts de la colonisation. Charlesbourg et Beauport, par exemple, sont de grosses paroisses bien organisées. Il en va tout autrement dans l'île d'Orléans où les longs délais restent relativement nombreux, en dépit d'une évolution rapide. Mais les facteurs climatiques n'y jouent pas un rôle déterminant, décembre se comparant aux mois d'été. De nombreux éléments du genre de vie, on le devine, favorisaient ces variations locales.

TABLEAU 10

Proportion de baptêmes à 3 jours et plus suivant l'habitat et le mois de naissance durant la période 1680-1699

\begin{tabular}{|c|c|c|c|c|c|}
\hline \multirow{3}{*}{$\begin{array}{c}\text { Mois de } \\
\text { naissance }\end{array}$} & \multicolumn{5}{|c|}{ Nombre de baptêmes à 3 jours et plus (pour 100) } \\
\hline & \multirow[t]{2}{*}{ Ville* } & \multicolumn{3}{|c|}{ Campagne } & \multirow[t]{2}{*}{ Ensemble } \\
\hline & & Total & $\begin{array}{l}\text { Environs de } \\
\text { Québec** }\end{array}$ & Île d'Orléans & \\
\hline Janvier & 3,3 & 27,8 & 7,7 & 32,7 & 21,7 \\
\hline Février & 3,3 & 25,4 & 3,9 . & 29,0 & 19,7 \\
\hline Mars & 3,1 & 24,6 & 3,9 & 29,9 & 19,6 \\
\hline Avril & 2,6 & 21,4 & 2,6 & 15,5 & 16,3 \\
\hline Mai & 9,3 & 22,4 & 1,5 & 28,0 & 18,4 \\
\hline Juin & 5,3 & 16,8 & 1,7 & 19,7 & 13,4 \\
\hline Juillet & 5,4 & 17,7 & 1,4 & 23,2 & 13,1 \\
\hline Août & 5,5 & 19,5 & 1,7 & 19,5 & 15,2 \\
\hline Septembre & 4,8 & 24,5 & 3,7 & 23,9 & 18,3 \\
\hline Octobre & 7,8 & 26,8 & 0,0 & 34,2 & 20,7 \\
\hline Novembre & 3,1 & 20,9 & 2,3 & 22,5 & 16,1 \\
\hline Décembre & 1,5 & 17,5 & 5,4 & 19,0 & 13,0 \\
\hline Ensemble & 4,6 & 22,4 & 3,2 & 25,2 & 17,3 \\
\hline $\begin{array}{l}\text { Nombres absolus } \\
\text { de baptisés } \\
\text { d'âge au } \\
\text { baptême connu }\end{array}$ & 2533 & 6280 & 901 & 992 & 8813 \\
\hline
\end{tabular}

* Québec et Montréal, à l'exclusion de Trois-Rivières.

** Sillery, Beauport, l'Ancienne-Lorette, Charlesbourg et Ste-Foy.

\section{Conclusion}

Ainsi la rudesse du pays et les insuffisances de l'organisation paroissiale dans la colonie naissante n'ont pas empêché l'observance de la réglementation ecclésiastique. La concentration du peuplement de part et d'autre de l'axe fluvial favorisait les communications et les prê- 
tres missionnaires ont déployé une activité exemplaire qui nous vaut aujourd'hui des documents de très grande valeur.

ANNEXE

Âge moyen et âge médian au baptême par paroisse avant 1700

\begin{tabular}{|c|c|c|c|c|}
\hline Paroisse & $\begin{array}{l}\text { Nombre total } \\
\text { de baptisés }\end{array}$ & $\begin{array}{c}\text { Âge au baptême } \\
\text { connu pour } 100 \\
\text { baptisés }\end{array}$ & $\begin{array}{l}\text { Âge moyen } \\
\text { au baptême } \\
\text { (en jours) }\end{array}$ & $\begin{array}{c}\text { Âge médian } \\
\text { au baptême } \\
\text { (en jours) }\end{array}$ \\
\hline Contrecoeur & 123 & 99,2 & 16,9 & 5,6 \\
\hline Neuville & 594 & 98,3 & 3,9 & 1,8 \\
\hline Boucherville & 724 & 95,6 & 4,6 & 1,6 \\
\hline Beauport & 434 & 95,6 & 1,2 & 1,1 \\
\hline Rivière-Ouelle & 155 & 95,5 & 16,3 & 5,7 \\
\hline Ste-Famille I.O. & 1050 & 94,7 & 4,4 & 2,0 \\
\hline Charlesbourg & 463 & 93,5 & 0,9 & 1,2 \\
\hline Repentigny & 146 & 93,2 & 4,3 & 1,8 \\
\hline Québec & 4631 & 91,3 & 5,3 & 1,4 \\
\hline Lauzon & 202 & 90,6 & 4,6 & 1,9 \\
\hline Lachine & 326 & 89,0 & 3,9 & 1,4 \\
\hline Château-Richer & 787 & 88,8 & 3,2 & 1,6 \\
\hline Cap-St-Ignace & 214 & 88,3 & 9,5 & 3,0 \\
\hline Ste-Anne-de-Beaupré & 313 & 87,5 & 4,0 & 1,5 \\
\hline L'Ange-Gardien & 362 & 87,8 & 2,4 & 1,7 \\
\hline St-Pierre I.O. & 273 & 85,0 & 3,2 & 2,0 \\
\hline St-Laurent I.O. & 283 & 78,4 & 3,3 & 1,9 \\
\hline Cap-de-la-Madeleine & 169 & 75,1 & 5,0 & 2,2 \\
\hline L'Ancienne-Lorette & 141 & 73,0 & 1,7 & 1,4 \\
\hline Laprairie & 315 & 70,2 & 1,3 & 1,2 \\
\hline St-François I.O. & 156 & 67,3 & 2,3 & 2,0 \\
\hline Sillery & 266 & 63,2 & 2,4 & 1,8 \\
\hline Champlain & 301 & 62,1 & 3,4 & 1,7 \\
\hline St-Jean I.O. & 224 & 59,4 & 3,1 & 1,7 \\
\hline Batiscan & 292 & 54,5 & 1,4 & 1,3 \\
\hline Sorel & 257 & 52,9 & 19,2 & 5,9 \\
\hline Trois-Rivières & 688 & 52,0 & 5,5 & 1,5 \\
\hline Pointe-aux-Trembles & 534 & 36,0 & 1,6 & 1,3 \\
\hline Montréal & 2528 & 33,7 & 4,7 & 0,8 \\
\hline \multicolumn{5}{|l|}{$\begin{array}{l}\text { Paroisses comptant } \\
\text { moins de } 100 \text { âges }\end{array}$} \\
\hline au baptême connus* & 775 & 79,6 & 7,0 & 2,0 \\
\hline Ensemble & 17726 & 76,3 & 4,7 & 1,5 \\
\hline
\end{tabular}

* La Pérade (87), Varennes (85), St-Michel (57), L’Islet (55), St-Thomas (55), Baie-St-Paul (47), Lachenaie (44), Beaumont (43), Grondines (34), St-Augustin (28), Cap-Santé (22), StNicolas (20), St-François-du-Lac (15), Ste-Foy (13), St-Joachim (5), Ste-Anne-de-Bellevue (3), Tadoussac (3), Lotbinière (1). 
L'étude de l'âge au baptême ne constitue cependant qu'un bien pâle reflet des richesses offertes par l'ensemble des registres paroissiaux du Québec ancien. Si la seule comparaison entre deux dates nous apporte quelques enseignements, on imagine sans peine ce que les divers spécialistes sont maintenant en mesure d'extraire de la banque de données du Programme de recherche en démographie historique. La reconstitution des familles complètement achevée pour la période antérieure à 1730 autorise les perspectives les plus impressionnantes. Désormais 100000 actes attendent, sous forme de fiches de famille et de généalogies, les multiples traitements informatiques que les chercheurs voudront leur faire subir. Même les projets en marche ne sont pas prêts d'épuiser un tel potentiel. 